\title{
Anat Feinberg, Wieder im Rampenlicht. Jüdische Rückkehrer in deutschen Theatern nach 1945
}

\author{
Laurence Guillon
}

\section{OpenEdition}

\section{Journals}

Édition électronique

URL : http://journals.openedition.org/ifha/9802

DOI : $10.4000 /$ ifha.9802

ISSN : 2198-8943

Éditeur

IFRA - Institut franco-allemand (sciences historiques et sociales)

Référence électronique

Laurence Guillon, «Anat Feinberg, Wieder im Rampenlicht. Jüdische Rückkehrer in deutschen Theatern nach 1945 », Revue de l'IFHA [En ligne], Date de recension, mis en ligne le 13 décembre 2018, consulté le 24 septembre 2020. URL : http://journals.openedition.org/ifha/9802 ; DOI : https://doi.org/10.4000/ ifha.9802

Ce document a été généré automatiquement le 24 septembre 2020.

(C)IFHA 


\section{Anat Feinberg, Wieder im Rampenlicht. Jüdische Rückkehrer in deutschen Theatern nach 1945}

Laurence Guillon

\section{RÉFÉRENCE}

Anat Feinberg, Wieder im Rampenlicht. Jüdische Rückkehrer in deutschen Theatern nach 1945, Göttingen : Wallstein Verlag, 2018, 336 p., $29 €$ 
Anat Feinberg, spécialiste israélienne de littérature juive et hébraïque à la Hochschule für jüdische Studien de Heidelberg, revient dans cet ouvrage à un thème qui lui a toujours été cher: le théâtre allemand après la Seconde Guerre mondiale. Après s'être intéressée il y a une dizaine d'années au destin des musiciens juifs en Allemagne après 1945, elle revient à celui des hommes et des femmes de théâtre qui choisirent envers et contre tout de se réinstaller et de recommencer une carrière en Allemagne après la Shoah. Qui dit "Allemagne", dit en réalité "Allemagnes », puisqu'en plus de faire le choix de revenir au "pays des meurtriers ", ces artistes ont dû se décider entre l'Est et l'Ouest. Et c'est précisément un des mérites de cette étude que d'adopter un regard global sur cette

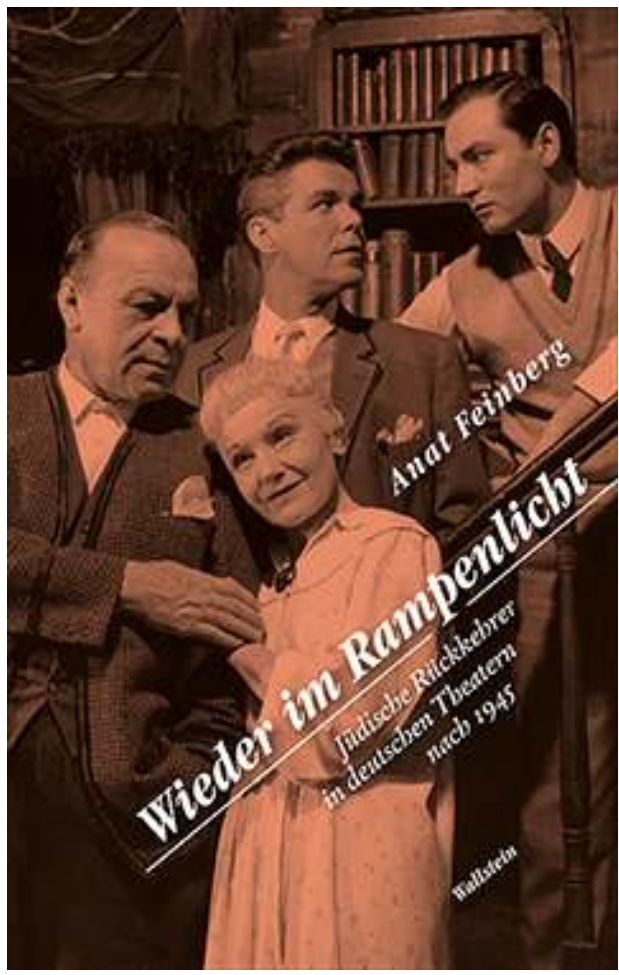
rémigration bien spécifique, permettant de nombreux aller-retours entre RDA et RFA, et participant ainsi de la vaste entreprise d'«histoire intégrée " (Christoph Kleßmann) qui peine encore s'imposer, mais s'avère très féconde sur le plan de l'histoire culturelle. En particulier, la liste des artistes rémigrés présentée en fin d'ouvrage, incluant la RDA et la RFA, constitue un outil extrêmement précieux pour les chercheurs qui s'intéressent à la vie culturelle dans l'Allemagne divisée.

Spécifique, la rémigration d'anciens acteurs et actrices, metteur(e)s en scène, cabarettistes, directeurs de théâtres, dramaturges, décorateurs et assistants techniques, l'est à plus d'un titre, mais sa singularité réside avant tout dans le fait que le premier matériau sur lequel ils travaillent, la langue, leur a été retiré durant les années d'exil. Il n'est donc pas surprenant que leur retour soit motivé pour beaucoup par le retour au pays de la langue maternelle (Sprachheimat pour Fritz Kortner). L'expérience de l'exil fut pour eux la pire des catastrophes, comme le résume très bien cette citation de l'acteur Ernst Deutsch : «À part une maladie incurable, l'émigration forcée est ce qui peut arriver de pire à une jeune personne. »

A. Feinberg s'intéresse aussi bien à celles et ceux qui se sont établis durablement en Allemagne qu'aux Sojourners qui y sont revenus pour un temps relativement long, mais sans s'y fixer définitivement. Il paraît également judicieux de distinguer, comme elle le fait, ces rémigrés persécutés pour raisons raciales de ceux qui l'ont été pour des raisons politiques. Son ouvrage contribue par ailleurs à "démocratiser » la recherche sur la rémigration en ne s'intéressant pas uniquement aux élites. Au total, ce sont environ 200 personnes qu'elle étudie, dont $28 \%$ de femmes. Dans la grande majorité des cas, celles et ceux qui ont fait le choix de s'établir en RDA (environ 15\%) et d'y travailler comme artistes y sont restés jusqu'à leur mort, ce qui est moins vrai pour ceux qui se sont établis en RFA. Toutes ces personnes revenaient de 25 pays différents, $42 \%$ ayant trouvé refuge aux États-Unis. 
Le principe directeur de l'étude est de lier intrinsèquement perspective individuelle et perspective collective. Après avoir dressé un portrait collectif des rémigrés dans le monde du théâtre, A. Feinberg s'intéresse à quatre destins, déjà bien explorés pour certains, comme ceux de Ernst Deutsch, Steffie Spira ou Herbert Grünbaum, ou tombés dans l'oubli comme celui de Claudius Kraushaar, directeur du Schauspielhaus de Stuttgart sous la République de Weimar. La richesse de cette présentation réside dans les archives en partie inédites sur lesquelles s'est appuyée l'auteure (Nachlässe, correspondances, documents administratifs).

Comme on le sait, il y eut en réalité très peu d'appels au retour de ces artistes de la part de la société allemande, si ce n'est par des rémigrés eux-mêmes comme Gustav von Wangenheim, intendant du Deutsches Theater, ancien exilé en URSS. Une exception notable à cet égard fut l'appel du secrétaire d'État Dieter Sattler en Bavière. Ceux qui semblent avoir le mieux réussi à se réintégrer dans les Allemagnes d'après-guerre sont finalement les "Reklame-Juden", ces grands noms d'artistes qui avaient marqué le monde du théâtre de la République de Weimar. Mais la plupart d'entre eux firent l'expérience d'une amère déception: la difficulté de se réimplanter en Allemagne fut incommensurable. Certains avaient dû changer de métier dans leur pays d'exil, devenant ouvrier agricole, apiculteur ou restaurateur... La déchéance du grand acteur Curt Bois, qui n'avait pas de quoi s'offrir la traversée de l'Atlantique pour rentrer au pays, est symptomatique. D'innombrables obstacles bureaucratiques se présentaient également, exacerbés par les tensions politiques entre Est et Ouest, notamment durant le blocus de Berlin. Par ailleurs, les acteurs avaient perdu tout contact avec la scène théâtrale allemande et leur jeu pouvait apparaître comme " archaïque ». L'influence du «style nazi » avait laissé des traces sur le jeu des acteurs et le goût du public. Mais surtout, les rémigrants furent confrontés à la persistance d'un antisémitisme ouvertement assumé, qui explique qu'un Max Colpet ait préféré la Suisse et qu'un Fritz Kortner ait passé le reste de sa vie à le dénoncer. L'amnésie du milieu du théâtre concernant l'histoire récente est déroutante. Le Juif était toujours perçu, selon les termes d'Eric Schildkraut, comme « le marginal, le concurrent, le Juif qui rappelait par sa présence ce qui était obstinément refoulé ». De nombreux acteurs très en vue avaient joué un rôle éminent sous le national-socialisme et ils voyaient maintenant d'un mauvais œil cette nouvelle concurrence arriver.

On peine peut-être parfois à trouver une ligne de force parmi la diversité des expériences individuelles, et certaines anecdotes se répètent dans différents développements, mais A. Feinberg met en valeur de façon admirable l'ambivalence des sentiments et des réactions de ces rémigrés, montrant l'impossibilité de renouer avec le pays des temps anciens - une situation face à laquelle Carl Zuckmayer était très lucide dès 1939 : «Je sais que je reverrai tout cela et que je ne retrouverai plus rien de ce que j'ai quitté. »

En conclusion, cet ouvrage vient enrichir le champ encore peu exploré il y a quelques années de la recherche sur l'exil, qui commence à s'étoffer dans le sillage de l'étude synoptique de Marita Krauss et des études sur des dimensions particulières du retour d'exil. 
INDEX

Index chronologique : Époque contemporaine

Thèmes : Histoire de la culture

\section{AUTEURS}

LAURENCE GUILLON

Université Lyon 2, EA 1853 